\title{
Entupimento de gotejadores e seu efeito na pressão da rede hidráulica de um sistema de microirrigação
}

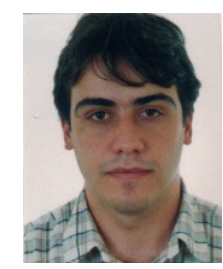

\author{
Luís F. Faria ${ }^{1}$, Rubens D. Coelho ${ }^{2}$, Pablo A. N. Flecha ${ }^{1}$, Walter G. R. Robles ${ }^{1}$ \& Manuel A. N. Vásquez ${ }^{1}$ \\ 1 ESALQ/USP. Av. Pádua Dias 11, CP 09, CEP 13418-900, Piracicaba, SP (Foto) \\ 2 Departamento de Engenharia Rural. E-mail: rdcoelho@carpa.ciagri.usp.br
}

Protocolo 133 - 19/9/2001

\begin{abstract}
Resumo: Com o presente trabalho, objetivou-se avaliar a sobreelevação de pressão na rede hidráulica de um sistema de irrigação por gotejamento, quando ocorre redução da vazão por entupimento dos emissores. A área avaliada foi de 280 ha, irrigando citrus através de gotejamento subsuperficial, com emissores autocompensantes. A metodologia consistiu num modelo matemático baseado na equação de Bernoulli, utilizando-se uma planilha eletrônica para simular os efeitos do entupimento dos emissores na malha hidráulica. Para estimar a variação de pressão no sistema, simulou-se obstrução dos emissores de 0 a 100\%. Para a condição de 50\% de entupimento, $80 \%$ do comprimento da malha hidráulica apresentaram pressão superior à da classe de pressão da tubulação. O modelo matemático permitiu simular: (a) a variação da rotação do conjunto moto-bomba diesel, como possível solução para controle da sobreelevação de pressão na malha hidráulica e (b) a variação de pressão na rede, devido ao excesso de rotação no motor diesel (imprecisão no tacômetro). Quando se simularam a situação de $50 \%$ de entupimento e 2100 rotações $\min ^{-1}$, 98\% do comprimento da tubulação apresentaram pressão acima à da classe de pressão da tubulação.
\end{abstract}

Palavras-chave: simulação, irrigação localizada, gotejamento, entupimento.

\section{Clogging of drippers and its effect on the hydraulic network pressure in a micro-irrigation system}

\begin{abstract}
The present study attempts to evaluate the pressure increase in the hydraulic network of a micro-irrigation system when partial or total clogging of emitters occurs. The evaluated area was of 280 ha, with citrus irrigation based on subsurface auto-compensating drippers. The methodology utilized a mathematical model based on Bernoulli's equation, simulating the effects of different clogging rates on the network pressure. To evaluate the pressure variation in the system, the obstructions in the range from 0 to $100 \%$ were simulated. For the condition of $50 \%$ clogging, $80 \%$ of the total pipeline length presented hydraulic pressure more than the nominal pressure foreseen in the project. The mathematical model permitted the simulation of: (a) the variation of the motor-speed of the pump (diesel energy), as a possible solution for controlling the excess pressure problem due to clogging (b) the pressure increase in the network with rotation in excess caused by tachometer imprecision or labor misconduct. When the clogging of $50 \%$ of the emitters was simulated with the motor-speed under 2100 rotation $\mathrm{min}^{-1}, 98 \%$ of the pipeline of the system developed pressures in excess of the normal range.
\end{abstract}

Key words: simulation, trickle irrigation, drip irrigation, plugging

\section{INTRODUÇÃO}

A irrigação localizada vem sendo largamente utilizada, tanto na forma de micro-aspersão como gotejamento; sua utilização pode proporcionar alta eficiência do uso da água, quando comparada com outros métodos, como aspersão convencional e irrigação por superfície. Tal fato possibilita maior precisão de aplicação de água, fertilizantes e defensivos via água de irrigação, além da possibilidade de aumento da área irrigada, devido às menores vazões demandadas pelo método. $\mathrm{O}$ efeito do entupimento dos emissores na malha hidráulica é um problema sério para a operação e manutenção de um sistema de irrigação por gotejamento, tendo levado muitos irrigantes a abandonarem precocemente o sistema (Ghaemi \& Chieneg, 1999).

Bralts et al. (1987) e Wu et al. (1988) identificaram cinco situações em um sistema de microirrigação na qual ocorre entupimento: (1) entupimento parcial, que afeta todos os emissores; (2) entupimento parcial, que afeta uma proporção específica dos emissores, de forma igual; (3) entupimento completo, afetando igualmente todos os emissores; (4) entupimento completo, afetando uma proporção específica dos 
emissores e (5) uma combinação de entupimento parcial e completo. A quinta situação é um caso geral e os outros podem ser dela derivados. Em situações de campo há, normalmente, alguns emissores parcial ou completamente entupidos.

Lau et al. (1978) relataram experiências de campo, nas quais mostraram que o entupimento é predominante na extremidade final da lateral. Wu et al. (1991) confirmaram que o entupimento não é distribuído uniformemente ao longo da linha lateral.

A baixa qualidade da água de irrigação pode obstruir rapidamente os emissores. Em geral, as causas do entupimento de emissores podem ser divididas em três categorias: (1) nos componentes físicos como areia e partículas de PVC; (2) nos materiais biológicos e (3) nas substâncias químicas precipitadas, como $\mathrm{CaCO}_{3}$. Podem ser eliminados os entupimentos físicos com o uso de sistemas de filtragem eficiente, os biológicos, com cloração e outros métodos de desinfecção, e a precipitação química com controle do $\mathrm{pH}$ da água de irrigação (Yuan et al., 1998).

Resende (1999) estudando o entupimento por causas biológicas, notou que em um dos modelos de gotejadores estudados ocorreu redução de vazão de $40 \%$ em $16 \%$ do número total de gotejadores, para $2.160 \mathrm{~h}$ de funcionamento. Em outro modelo, ele observou $20 \%$ de redução de vazão em $24 \%$ dos emissores do modelo para $1.200 \mathrm{~h}$ de funcionamento.

Outras causas de entupimento dos gotejadores estão ligadas à sucção de partículas sólidas e à intrusão radicular em sistemas subsuperficiais (Pizarro Cabello, 1996).

Estudos enfocando os danos ocasionados à tubulação de distribuição de água e ao sistema de bombeamento são ainda escassos. A redução da vazão dos emissores provoca redução da velocidade da água na tubulação, reduzindo as perdas de carga por atrito. Outro fato importante é a alteração do ponto de funcionamento do sistema moto-bomba, fato este que incrementa a pressão de saída na bomba. Não raro, os operadores de campo dos sistemas de irrigação provocam incrementos na rotação do conjunto moto-bomba, acreditando que isto atue de maneira eficaz para a recuperação da vazão dos emissores, ou simplesmente por descuido. A combinação dos eventos relatados anteriormente, proporciona sobreelevação de pressão na tubulação de distribuição de água nos sistemas de microirrigação, comprometendo a rede hidráulica e reduzindo sua vida útil. Uma medida possível e paliativa seria a redução da rotação da moto-bomba, facilmente controlada em motores de combustão interna. Tendo em vista os fatos relatados, este trabalho tem, como objetivo: 1) estudar a sobreelevação de pressão na rede hidráulica, devido ao entupimento dos emissores; 2) simulação da redução de rotação do conjunto moto-bomba e 3 ) simulação de aumento de rotação do conjunto moto-bomba.

\section{MATERIAL E MÉTODOS}

Para a elaboração do presente estudo desenvolveu-se uma planilha eletrônica (Excel) com ferramentas do recurso macro. Utilizou-se um projeto de irrigação por gotejamento, elaborado para irrigar citrus, com as seguintes características: área irrigada de 280 ha, vazão nominal dos gotejadores de $3,6 \mathrm{~L} \mathrm{~h}^{-1}$ autocompensantes, espaçamento entre emissores de 1,0 m, com 2 linhas de gotejadores por linha de plantas, acionado por duas bombas diesel em paralelo (bomba multiestágio) com potência de $100 \mathrm{~kW}$.
O sistema de irrigação foi projetado com 60 subunidades de irrigação, operando em 6 setores, cada qual com 10 subunidades. Na rede hidráulica de PVC foram identificados 47 trechos, com comprimento variando de 44 a 720 m, diâmetros nominais entre 100 e $250 \mathrm{~mm}$, classe de pressão nominal entre 400 e $1250 \mathrm{kPa}$, resultando o funcionamento de cada um dos setores com combinações diferentes desses trechos.

A curva vazão versus pressão fornecida pelo conjunto motobomba e a equação de ajuste, são apresentadas na Figura 1.

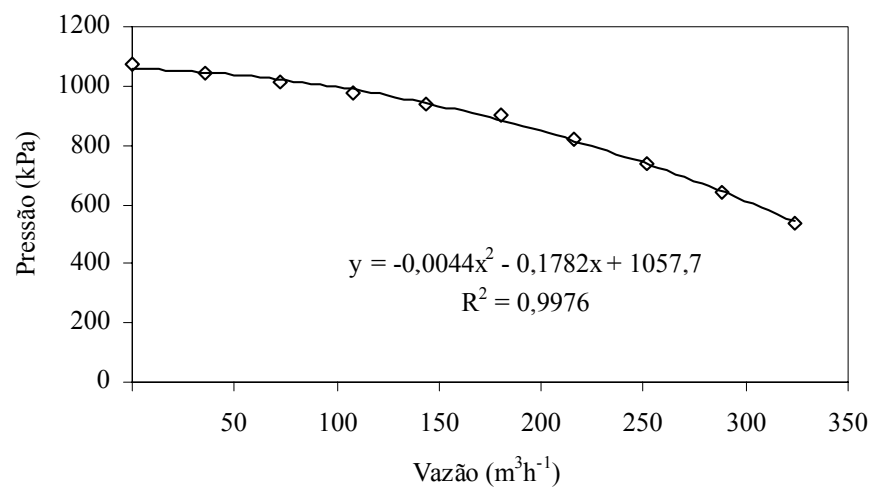

Figura 1. Curva característica da bomba

As perdas de carga da tubulação foram determinadas pela equação de Hazen-Williams. A pressão na entrada e saída de cada trecho foi obtida pela equação de Bernoulli, ou seja, contabilização de pressão fornecida pelo trecho subseqüente, perda de carga por atrito e diferença de nível topográfico. $\mathrm{O}$ comprimento de cada trecho foi determinado por derivações de água à montante e à jusante e pela especificação da tubulação, no que se refere ao diâmetro nominal e classe de pressão.

Os recursos utilizados na planilha possibilitam a redução uniforme de vazão dos gotejadores, de 0 a 100\%, e a variação de rotação do conjunto moto-bomba de 1.450 a 2.100 rotações min ${ }^{-1}$, sendo que a pressão fornecida pela bomba é variável de acordo com a rotação e a vazão do sistema. Para se obter a pressão fornecida pela bomba a uma rotação diferente da original, dividiuse a pressão original por um fator (f) obtido pela expressão:

$$
\mathrm{f}=\left(\frac{\operatorname{rotação} o_{1}}{\operatorname{rotação} o_{2}}\right)^{2}
$$

em que:

f - fator divisor da pressão original, adimensional rotação - rotação original da bomba, rotações $\min ^{-1}$ rotação ${ }_{2}$ - rotação atual da bomba, rotações $\min ^{-1}$

Os resultados apresentados neste trabalho referem-se a intervalo de classe de $10 \%$ e amplitude de 0 até $100 \%$ para o entupimento e rotação com intervalo de 50 rotações min $^{-1} \mathrm{e}$ amplitude de 1450 a 2100 rotações $\mathrm{min}^{-1}$. As simulações foram realizadas com todos os setores do projeto.

\section{RESULTADOS E DISCUSSÃO}

As respostas ao entupimento e à variação de rotação do sistema moto-bomba, mostraram-se semelhantes nos 6 setores do sistema de irrigação em questão, tornando-se desnecessário 
a apresentação dos resultados para todos eles. Tendo em vista este fato, aqui são apresentados os resultados para um setor operação.

As Figuras 2A, B e C mostram, respectivamente, as situações de operação: emissores com vazão nominal e conjunto moto-bomba, operando a 1.750 rotações $\mathrm{min}^{-1}$; emissores com $50 \%$ de entupimento e moto-bomba, operando a 1.750 rotações min $^{-1}$; emissores com $50 \%$ de entupimento e moto-bomba, operando a 1.500 rotações $\mathrm{min}^{-1}$.
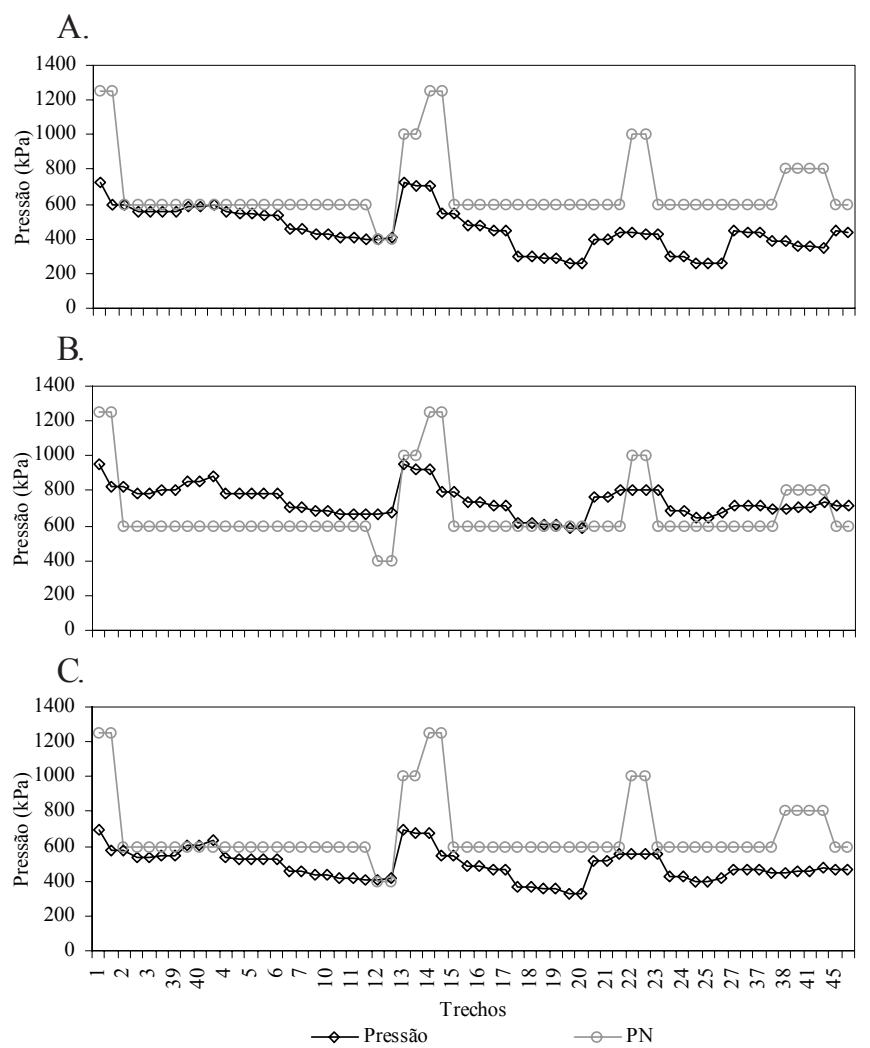

Figura 2. Emissores a vazão nominal e moto-bomba 1.750 rotações $\min ^{-1}$ (A), a 50\% de entupimento e moto-bomba a 1.750 rotações $\min ^{-1}(\mathrm{~B})$ e a $50 \%$ de entupimento e moto-bomba a 1.500 rotações $\min ^{-1}(\mathrm{C})$

Nessas figuras, os trechos identificados no eixo das abscissas representam, de maneira seqüencial, o seguimento de tubulação, não indicando que a seqüência seja a que ocorre na distribuição da água, pois a rede é dotada de muitas ramificações. No eixo das ordenadas são apresentadas as pressões atual e nominal da tubulação.

Pela Figura 2A, pode-se notar que o sistema funcionando à vazão nominal, não apresenta trechos operando com sobreelevação de pressão, ou seja, a pressão máxima dentro de cada trecho não ultrapassa a classe de pressão da tubulação, indicando que o projeto hidráulico foi criteriosamente dimensionado no que se refere à pressão dinâmica.

Já na Figura 2B, situação de operação dos emissores com $50 \%$ de redução de vazão e rotação original do sistema motobomba, constata-se que em vários trechos houve grande incremento de pressão. Quando se simulou a redução de rotação do sistema moto-bomba, verificou-se redução da pressão na malha hidráulica, estando o sistema em situação semelhante à condição original de operação (Figura 2C).
Como se observa na Figura 3A, onde os emissores estão à vazão nominal e a rotação do conjunto moto-bomba está a 2100 rotações min $^{-1}$, grande parte da rede hidráulica do sistema apresenta pressão atual acima da pressão nominal do sistema. A Figura 3B mostra 50\% de redução de vazão dos emissores e 2100 rotações $\min ^{-1}$ na moto-bomba. Nesta situação, praticamente toda a rede hidráulica do sistema se apresenta comprometida, a níveis elevados de sobreelevação de pressão.
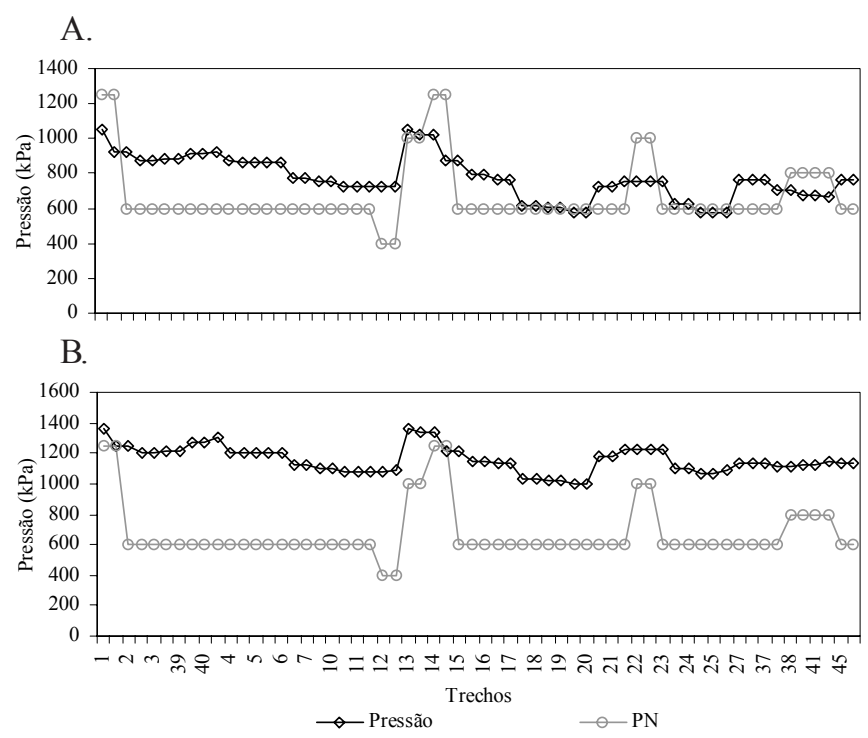

Figura 3. Emissores a vazão nominal e moto bomba a 2100 rotações $\min ^{-1}(\mathrm{~A})$ e a $50 \%$ de entupimento e moto-bomba a 2100 rotações $\min ^{-1}(\mathrm{~B})$

A Tabela 1 apresenta o resumo do grau de entupimento dos emissores e os comprimentos de tubulação (m) com-prometidos em categorias de sobreelevação de pressão na extensão total da rede $(4.500 \mathrm{~m})$. Essas categorias indicam acréscimos de pressão em função da classe de pressão da tubulação, ou seja, na categoria de zero até 10 , tem-se o comprimento dos trechos em metro, em condição de operação de até $10 \%$ de sobreelevação de pressão. A coluna (Total \%) mostra o percentual em relação ao comprimento da rede hidráulica, comprometida em qualquer nível de sobreelevação de pressão. Supondo-se que toda a malha hidráulica seja composta apenas por tubos da classe de pressão $(\mathrm{PN}) 400 \mathrm{kPa}$, os trechos compreendidos nessa categoria estariam operando em pressão entre 400 e $440 \mathrm{kPa}$. Nota-se, na mesma tabela, que quando há $50 \%$ de entupimento, $80 \%$ da malha hidráulica se apresentam com sobreelevação de pressão.

A Tabela 2 apresenta o resumo do comportamento da pressão em função da rotação do sistema moto-bomba para emissores com $50 \%$ de redução de vazão e o comprimento dos trechos funcionando em categorias de sobreelevação de pressão em função da variação de rotação da moto-bomba.

Ainda na Tabela 2, constata-se que, com a redução da rotação, o comprimento de tubulação comprometido decresce até níveis próximos da condição original de funcionamento do sistema, quando a rotação atinge 1.500 rotações $\mathrm{min}^{-1}$.

A redução de rotação do sistema de bombeamento não deve ser admitida como solução definitiva para o problema de sobre-elevação de pressão, tendo em vista que o sistema de irrigação é projetado para uma demanda de água pelas plantas, 
Tabela 1. Entupimento dos gotejadores e comprimento dos trechos $(\mathrm{m})$ comprometidos em categorias de sobre elevação

\begin{tabular}{ccccccccccccc}
\hline \multirow{2}{*}{ Ent.\% } & \multicolumn{10}{c}{ Categorias de Sobre-Elevação de Pressão - \% } \\
\cline { 2 - 27 } & $0-10$ & $10-20$ & $20-30$ & $30-40$ & $40-50$ & $50-60$ & $60-70$ & $70-80$ & $80-90$ & $90-100$ & Total \\
\hline 10 & 810 & 300 & 0 & 0 & 0 & 0 & 0 & 0 & 0 & 0 & 19 \\
20 & 910 & 180 & 120 & 0 & 0 & 0 & 0 & 0 & 0 & 0 & 20 \\
30 & 1149 & 1065 & 360 & 180 & 120 & 0 & 0 & 0 & 0 & 0 & 48 \\
40 & 1514 & 1324 & 880 & 380 & 0 & 120 & 0 & 0 & 0 & 0 & 70 \\
50 & 279 & 2379 & 824 & 885 & 180 & 0 & 120 & 0 & 0 & 0 & 78 \\
60 & 168 & 1029 & 2074 & 1020 & 200 & 180 & 0 & 120 & 0 & 0 & 80 \\
70 & 245 & 168 & 2614 & 1194 & 515 & 180 & 0 & 0 & 120 & 0 & 84 \\
80 & 601 & 168 & 794 & 2309 & 945 & 455 & 0 & 0 & 120 & 0 & 90 \\
90 & 601 & 124 & 668 & 2479 & 945 & 275 & 180 & 0 & 0 & 120 & 90 \\
100 & 601 & 0 & 448 & 2478 & 1290 & 275 & 180 & 0 & 0 & 120 & 90 \\
\hline
\end{tabular}

Tabela 2. Rotação do conjunto moto-bomba e comprimento dos trechos (m) comprometidos em categorias de sobre-elevação para $50 \%$ de entupimento

\begin{tabular}{|c|c|c|c|c|c|c|c|c|c|c|c|c|}
\hline \multirow{2}{*}{ Rot. } & \multicolumn{12}{|c|}{ Categorias de Sobre-Elevação de Pressão - \% } \\
\hline & $0-10$ & $10-20$ & $20-30$ & $30-40$ & $40-50$ & $50-60$ & $60-70$ & $70-80$ & $80-90$ & $90-100$ & $>100$ & Total \\
\hline 2100 & 534 & 0 & 72 & 356 & 245 & 0 & 124 & 279 & 2379 & 839 & 1030 & 98 \\
\hline 2050 & 0 & 72 & 126 & 475 & 0 & 124 & 898 & 1904 & 1050 & 440 & 300 & 90 \\
\hline 2000 & 72 & 0 & 601 & 0 & 124 & 898 & 1904 & 1125 & 440 & 180 & 120 & 91 \\
\hline 1950 & 72 & 601 & 0 & 124 & 898 & 1904 & 1125 & 440 & 180 & 0 & 120 & 91 \\
\hline 1900 & 230 & 371 & 124 & 898 & 1904 & 1125 & 440 & 180 & 0 & 0 & 120 & 90 \\
\hline 1850 & 780 & 124 & 898 & 1904 & 1125 & 440 & 180 & 0 & 0 & 120 & 0 & 93 \\
\hline 1800 & 124 & 384 & 2418 & 1050 & 515 & 180 & 0 & 0 & 120 & 0 & 0 & 80 \\
\hline 1750 & 279 & 2379 & 824 & 885 & 180 & 0 & 120 & 0 & 0 & 0 & 0 & 78 \\
\hline 1700 & 2219 & 1219 & 885 & 180 & 0 & 120 & 0 & 0 & 0 & 0 & 0 & 77 \\
\hline 1650 & 1324 & 880 & 380 & 0 & 120 & 0 & 0 & 0 & 0 & 0 & 0 & 45 \\
\hline 1600 & 880 & 380 & 120 & 0 & 0 & 0 & 0 & 0 & 0 & 0 & 0 & 23 \\
\hline 1550 & 360 & 300 & 0 & 0 & 0 & 0 & 0 & 0 & 0 & 0 & 0 & 11 \\
\hline 1500 & 2 & 0 & 0 & 0 & 0 & 0 & 0 & 0 & 0 & 0 & 0 & 0 \\
\hline 1450 & 0 & 0 & 0 & 0 & 0 & 0 & 0 & 0 & 0 & 0 & 0 & 0 \\
\hline
\end{tabular}

e quando ocorre redução de vazão dos gotejadores, esta demanda pode não estar sendo suprida, pois o sistema dimensionado por critérios econômicos admite pouca folga no turno de irrigação. Uma medida para melhor solução do problema não envolvendo a substituição das linhas laterais, seria o redimensionamento desse sistema, procurando-se novas combinações de operação das subunidades de funcionamento e se aumentando o número de subunidades por setor de operação diminuindo, conseqüentemente o número de setores possibilitando, assim, maior tempo disponível por setor.

A presente simulação não levou em consideração a existência de acessórios como válvula de segurança e alívio, que poderiam agir quando ocorresse sobreelevação de pressão. Tal consideração não foi realizada porque esses dispositivos são dimensionados para uso eventual e as condições simuladas têm duração prolongada.

\section{CONCLUSÕES}

1. A redução de vazão dos emissores provocou aumento considerável da pressão na tubulação de distribuição de água do sistema em estudo.

2. A redução de rotação do sistema moto bomba mostrouse como medida eficiente da redução de pressão na malha hidráulica do sistema.

3. O aumento de rotação do conjunto moto-bomba, tanto para o sistema operando com os gotejadores em sua vazão nominal como com redução de vazão de $50 \%$, apresenta acréscimos excessivos à pressão na rede hidráulica do sistema.

\section{LITERATURA CITADA}

Bralts, V. F.; Edwards D. M.; Wu, I. P. Drip irrigation design and evaluation based on the statistical uniformity concept. Advances in Irrigation, New York, p.67-117. 1987.

Ghaemi, A.; Chieng, S. Emitter clogging and hydraulics in microirrigation lines. In: Annual International Meeting, 1999, ASAE/CSAE-SCGR, Toronto, p. 8.

Lau, L. S.; Young, R. H. F.; Loh, P. C.; Bralts, V. F.; Liu, E. F. F. Recycling of sewage effluent by sugarcane irrigation: A post treatment study. Manoa, Honolulu: Water Resources Research Center, University of Hawaii. 1978. 42p. Technical Report No. 212

Pizarro Cabello, F. Riegos localizados de alta frecuencia. 3 ed. Madrid: Mundi-Prensa, 1996.513p.

Resende, R.S. Suscetibilidade de gotejadores ao entupimento de causa biológica e avaliação do desentupimento via cloração da água de irrigação. Piracicaba: ESALQ/USP, 1999. 77p. Dissertação Mestrado

Wu, I. P.; Irudayaraj, J. M.; Feng, J. S. Plugging evaluation for a drip irrigation submain unit. ASAE Paper No. 88-2062. St. Joseph: ASAE. 1988. 9p.

Wu, I. P.; Lin O. Y.; Lau, L. S. Plugging evaluation in the re-use of sewage effluent by drip irrigation. In: Irrigation and Drainage - Proceeding ASCE Speciality Conference, Honolulu, Hawaii: ASCE. 1991.p.780-786.

Yuan, Z.; Waller, P. M.; Choi, C. Y. Effects of organic acids on salt precipitation in drip emitters and soil. Transactions of the ASAE, St, Joseph. v.41, n.6, p.1689-1696, 1998. 